\title{
EVALUATION OF THE STABILITY OF THE PARAMETRIC PHASE NUMBER CONVERTER
}

\author{
Ivan Bedritskiy ${ }^{1 *}$, Kamila Jurayeva $^{1}$, Laziz Bazarov ${ }^{1}$ \\ ${ }^{1}$ Tashkent state transport university, Department of Power Supply, Adilkhodjayev - 1, Tashkent, 100167, Uzbekistan
}

\begin{abstract}
In the article related issues to the stability assessment of a parametric converter of the number of phases of a ferroresonance nature are analyzed. The areas of change in the characteristic parameters of the converter that guarantee its stable operation are considered.
\end{abstract}

\section{Introduction}

Artificial phase number converters are often used to power automation devices, computer equipment, and radio-electronic equipment, communications equipment, and electrical engineering devices. They are used in cases where the use of multiphase systems meets technical or economic difficulties[1, 2, 3, 4]. The most common devices of this type are three-phase-singlephase converters (so-called balancing devices) and single-phase-three-phase converters, which are used in single-phase circuits for powering three-phase consumers. At the same time, devices based on nonlinear

\section{Stateman of a problem}

Consider the parametric converter phases according to the scheme in Fig. 1 [9], where: $S_{1}, S_{2}, S_{3}$ square section rods of the magnetic core; $L_{1}, L_{2}, L_{3}$ - the average length of the magnetic lines of the magnetic circuit; $\varphi_{1}, \varphi_{2}, \varphi_{3}$ - instantaneous values of magnetic flux respectively in the cores of the magnetic circuit; $g_{1}, g_{2}-$ active conduction of the primary windings $W_{1}$ and $W_{2}$; $W_{1}, W_{2}$ - number of turns of the primary windings; $i_{1}, i_{2}$ - instantaneous value of current in the primary windings; $i_{g 1}, i_{g 2}$ - instantaneous value of current in the Taking into account the approximation of the magnetization curve $H=k \cdot b^{9}$, after some transformations, this scheme is described by a system of equations parametric LC circuits are the most acceptable for powering low-and medium-power consumers $[1,5,6,7$, $8]$, having high reliability, relatively low dimensions and weight.

However, devices based on parametric LC circuits can operate in the ferroresonance mode, so they may experience voltage surges (currents). Therefore, it is of interest to study the stability of parametric phase number converters and determine the boundaries of its stable operation.

transmissibility of primary windings; $C_{1}, C_{2}$ capacitors, connected in parallel with the primary windings $W_{1}$ and $W_{2}, i_{c 1}, i_{c 2}$ is the instantaneous current in the capacitors; $\quad i=I_{m} \cdot \sin \left(\omega t+\psi_{i}\right)$ is the instantaneous supply current; $u=U_{m} \cdot \sin \left(\omega t+\psi_{u}\right)$ is the instantaneous value of the supply voltage; $W_{3}, W_{4}, W_{5}$ are numbers of turns of the secondary winding of the transducer; $Z_{1}, Z_{2}, Z_{3}$ - resistance phase of three-phase load of the consumer; $U_{1 m}, U_{2 m}, U_{3 m}$ - voltage artificial phases.

$$
\left\{\begin{array}{l}
b_{1} \cdot S_{1}+b_{2} \cdot S_{2}-b_{3} \cdot S_{3}=0 \\
b_{1}{ }^{9}=\frac{k \cdot L_{2} \cdot b_{2}{ }^{9}-i_{2} \cdot W_{2}+i_{1} \cdot W_{1}}{k \cdot L_{1}} \\
u=W_{1} \cdot S_{1} \frac{d b_{1}}{d t}+W_{2} \cdot S_{2} \frac{d b_{2}}{d t} \\
i=i_{2}+i_{C}+i_{g 2} \\
i=i_{1}+i_{g 1}
\end{array}\right\},
$$




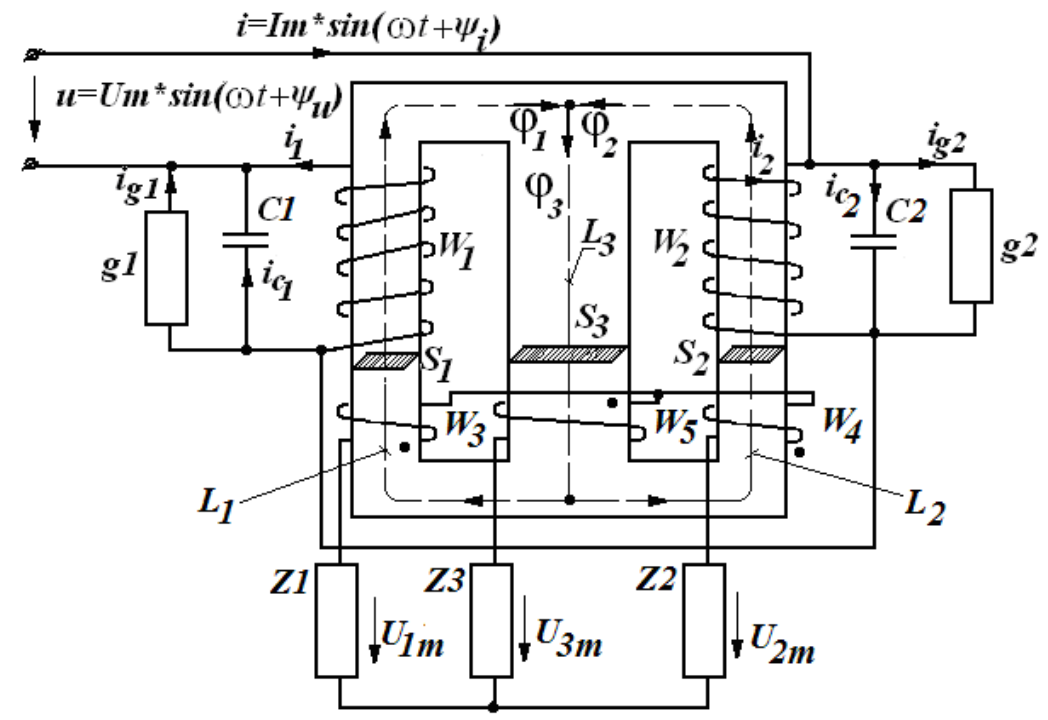

Fig. 1. Parametric phase number converter

where the instantaneous values of the currents in the branches of the circuit can be found from the expressions

$$
\begin{array}{lr}
i_{2}=\frac{k \cdot L_{2} \cdot b_{2}^{9}}{W_{2}} ; & i_{C}=W_{2} \cdot C \cdot S_{2} \cdot \frac{d_{2} b_{2}}{d t^{2}} ; \\
i_{g 2}=W_{2} \cdot g_{2} \cdot S_{2} \frac{d b_{2}}{d t} ; & i_{1}=\frac{k \cdot L_{1} \cdot b_{1}{ }^{9}}{W_{1}} ;
\end{array}
$$$$
i_{g 1}=W_{1} \cdot g_{1} \cdot S_{1} \cdot \frac{d b_{1}}{d t} \text {. }
$$$$
\begin{aligned}
& \frac{d F}{d t}=b_{1}^{9}\left(1+\frac{W_{1}}{W_{2}}\right)-b_{2}^{9}\left(\frac{L_{2}}{L_{1}}+\frac{W_{1} \cdot L_{2}}{W_{2}+L_{1}}\right)+\frac{d b_{1}}{d t}\left(\frac{W_{1}^{\prime} \cdot W_{2} \cdot g_{1} \cdot S_{1}+W_{1}^{2} \cdot g_{1} \cdot S_{1}}{k \cdot L_{1}}\right)- \\
& -F\left(\frac{g_{2} \cdot S_{2} \cdot W_{2}^{2}+W_{1} \cdot W_{2} \cdot g_{2} \cdot S_{2}}{k \cdot L_{1}}\right)
\end{aligned}
$$

Let's convert the system (1) to the form required for calculation on the computer, for which we will replace the variables

$$
\frac{d b_{2}}{d t}=F ; \quad \frac{d_{2} b_{2}}{d t^{2}}=\frac{d F}{d t} .
$$

Express it using the resulting expression $\frac{d F}{d t}$ and after conversion we get

Let's introduce the notation:

Express $\frac{d b_{1}}{d t}$ in accordance with (1), we get

$$
\begin{aligned}
& \frac{d b_{1}}{d t}=\frac{u-W_{2} \cdot S_{2} \cdot F}{W_{1} \cdot S_{1}} . \\
& \qquad A=W_{2} \cdot S_{2} ; B=W_{1} \cdot S_{1} ; \\
& \qquad M=\frac{W_{2}^{2} \cdot g_{2} \cdot S_{2}+W_{1} \cdot W_{2}}{k \cdot L_{1}} \\
& \text { Taking into account the accepted notation, } \\
& \text { expression for } \frac{d F}{d t} \quad \\
& \frac{d F}{d t}=\frac{b_{1}^{9} \cdot D-b_{2}^{9} \cdot k+\left(\frac{u-A \cdot F}{D} \cdot L\right)-F \cdot M}{N} .
\end{aligned}
$$

$$
\begin{aligned}
& A=W_{2} \cdot S_{2} ; B=W_{1} \cdot S_{1} ; D=1+\frac{W_{2}}{W_{1}} ; K=\frac{L_{2}}{L_{1}}+\frac{W_{1} \cdot L_{2}}{W_{2} \cdot L_{1}} ; L=\frac{W_{1} \cdot W_{2} g_{1} \cdot S_{1}+W_{1}^{2} \cdot g_{1} \cdot S_{1}}{k \cdot L_{1}} ; \\
& M=\frac{W_{2}^{2} \cdot g_{2} \cdot S_{2}+W_{1} \cdot W_{2} \cdot g_{2} \cdot S_{2}}{k \cdot L_{1}} ; N=\frac{W_{2}^{2} \cdot C \cdot S_{2}+W_{1} \cdot W_{2} \cdot C \cdot S_{2}}{k \cdot L_{1}}
\end{aligned}
$$

Taking into account the accepted notation, we get the

Given that $u=U_{m} \cdot \sin \left(\omega t+\psi_{u}\right)$, the expression (1) is converted to the form

This system of differential equations is used as a basis for studying the stability of the operation of a parametric phase number converter using the Hurwitz method [10, $11]$. 


\section{The concept of the problem decision}

We find general expressions for the study of the stability of a parametric phase number converter using elements of the stability theory. A system of differential equations (6) describing the electrical state of a circuit is known. To reduce the number of unknowns, we express $F$ from (2) and substituting it in (6) after the transformation, we get

$\left\{\begin{array}{l}\frac{d b_{1}}{d t}=A_{1} \cdot U_{m} \sin \left(\omega t+\psi_{u}\right)-A_{2} \cdot \frac{d b_{2}}{d t} \\ \frac{d_{2} b_{2}}{d t^{2}}=D_{1} \cdot b_{1}^{9}-D_{2} \cdot b_{2}^{9}+D_{3} \cdot U_{m} \sin \left(\omega t+\psi_{u}\right)-D_{4} \cdot \frac{d b_{2}}{d t}\end{array}\right\}$

where $\quad A_{1}=\frac{1}{B} ; \quad A_{2}=\frac{A}{B} ; \quad D_{1}=\frac{D}{N} ; \quad D_{2}=\frac{K}{N} ;$

$$
D_{3}=\frac{L}{B N} ; D_{4}=\frac{A L}{B N}-\frac{M}{N} \text {. }
$$$$
\left\{\begin{array}{l}
x \omega \cdot \cos \omega t-y \omega \cdot \sin \omega t=A_{1} \cdot U_{m} \sin \left(\omega t+\psi_{u}\right)-A_{2}(m \omega \cdot \cos \omega t-n \omega \cdot \sin \omega t) \\
-\left(\omega^{2} m+2 \omega \cdot \frac{d n}{d t}\right) \sin \omega t+\left(-\omega^{2} n+2 \omega \cdot \frac{d m}{d t}\right) \cos \omega t=D_{1}(x \sin \omega t+y \cos \omega t)^{9}- \\
-\left(D_{2}(m \sin \omega t+n \cos \omega t)^{9}+D_{3}\left(U_{m} \sin \left(\omega t+\psi_{u}\right)-D_{4}(m \omega \cdot \cos \omega t-n \omega \cdot \sin \omega t\right.\right.
\end{array}\right\}
$$

Transform (7) using the method of slowly varying amplitudes, assuming that the unknown $b_{1}, b_{2}$ can be represented as follows

$b_{1}=x \sin \omega t+y \cos \omega t$

$b_{2}=m \sin \omega t+n \cos \omega t$

where $x, y, m, n$ are the amplitudes of the desired oscillations that change slowly over time. We find the derivatives and given that $x, y, m, n$ change slowly over time, we get the first approximation

$\left\{\begin{array}{l}\frac{d b_{1}}{d t}=x \omega \cdot \cos \omega t-y \omega \cdot \sin \omega t \\ \frac{d b_{2}}{d t}=m \omega \cdot \cos \omega t-n \omega \cdot \sin \omega t \\ \frac{d_{2} b_{2}}{d t^{2}}=-\left(\omega^{2} m+2 \omega \cdot \frac{d n}{d t}\right) \sin \omega t+\left(-\omega^{2} n+2 \omega \cdot \frac{d m}{d t}\right) \cos \omega t\end{array}\right\}$

Substituting (9) in (7) we get
Transform the resulting expressions using the harmonic balance method. For the first expression, we have

$$
x \omega \cdot \cos \omega t-y \omega \cdot \sin \omega t=A_{1} U_{m} \sin \omega t \cdot \cos \psi_{u}+
$$$$
+A_{1} U_{m} \cos \omega t \cdot \sin \psi_{u}-A_{2} \omega \cos \omega t+A_{2} n \omega \cdot \sin \omega t
$$

Equating in (10) the coefficients for $\cos \omega t$ and sin $\omega t$ we get

$$
\begin{aligned}
& -y \omega=A_{1} U_{m} \cdot \cos \psi_{u}+A_{2} n \omega \\
& x \omega=A_{1} U_{m} \cdot \sin \psi_{u}-A_{2} m \omega
\end{aligned}
$$

Let us denote the coefficient for sin $\omega t$ in (12) by $X$, for $\cos \omega t$ by $Y$, and in (13) the coefficient for sin $\omega t$ by $M$, and for cos $\omega t$ by $N$. Taking into account the above

$$
\left\{\begin{array}{l}
-\left(\omega^{2} m+2 \omega \frac{d n}{d t}\right)=D_{1} X-D_{2} M+D_{3} U_{m} \cos \psi_{u}+D_{4} n \omega \\
-\omega^{2} n+2 \omega \frac{d m}{d t}=D_{1} Y-D_{2} N+D_{3} U_{m} \sin \psi_{u}-D_{4} m \omega
\end{array}\right\} .
$$

Taking into account (10) and (11), we have a system of algebraic and differential equations for relatively slowly varying amplitudes

$$
\left\{\begin{array}{l}
-y \omega=A_{1} U_{m} \cos \psi_{u}+A_{2} n \omega \\
x \omega=A_{1} U_{m} \sin \psi_{u}-A_{2} m \omega \\
-\left(\omega^{2} m+2 \omega \frac{d n}{d t}\right)=D_{1} X-D_{2} M+D_{3} U_{m} \cos \psi_{u}+D_{4} n \omega \\
-\omega^{2} n+2 \omega \frac{d m}{d t}=D_{1} Y-D_{2} N+D_{3} U_{m} \sin \psi_{u}-D_{4} m \omega
\end{array}\right\} .
$$

Transform the system (15) with respect to the derivatives of slowly varying amplitudes, for which we express $y$ and $x$ from the first two expressions (15), respectively. Receive

$$
y=\frac{A_{1} U_{m} \cos \psi-A_{2} n}{\omega} ; x=\frac{A_{1} U_{m} \sin \psi_{u}-A_{2} m}{\omega} .
$$

notation and comparing the coefficients for sinwt and cos $\omega t$, we transform the resulting expression using the harmonic balance method, we get the system

Denote $C=\frac{A_{1} U_{m} \cdot \cos \psi_{u}}{\omega} ; S=\frac{A_{1} U_{m} \cdot \sin \psi_{u}}{\omega}$.

Substitute (16) in the last two expressions of (15), taking into account the newly adopted notation. Since $X$ and $Y$ are functions of $x$ and $y$, with the new substitution they will become functions of $m$ and $n$. Receive 


$$
\begin{aligned}
& X_{m n}=0,5\left(s-A_{2 m}\right)^{9}+19,7\left(s-A_{2 m}\right)^{7}\left(-c-A_{2 n}\right)^{2}+29,5\left(s-A_{2 m}\right)^{5}\left(-c-A_{2 n}\right)^{4}+ \\
& +19,7\left(s-A_{2 m}\right)^{3}\left(-c-A_{2 n}\right)^{6}+2,5\left(s-A_{2 m}\right)\left(-c-A_{2 n}\right)^{8} \\
& Y_{m n}=14,8\left(s-A_{2 m}\right)^{8}\left(-c-A_{2 n}\right)+39,4\left(s-A_{2 m}\right)^{6}\left(-c-A_{2 n}\right)^{3}+ \\
& +29,5\left(s-A_{2 m}\right)^{4}\left(-c-A_{2 n}\right)^{5}+9,8\left(s-A_{2 m}\right)^{2}\left(-c-A_{2 n}\right)^{7}+0,5\left(-c-A_{2 n}\right)^{9} .
\end{aligned}
$$

By substituting (17) and (18) into the two lower equations of the system (15) after transformations, we

obtain a system of differential equations with respect to derivatives of slowly varying amplitudes $m$ and $n$ :

$$
\left\{\begin{array}{l}
\frac{d n}{d t}=\frac{-D_{1} X_{m n}+D_{2} M-D_{3} U_{m} \cos \psi_{u}}{2 \omega}-\frac{D_{4} \cdot n-\omega m}{2} \\
\frac{d m}{d t}=\frac{D_{1} Y_{m n}-D_{2} N+D_{3} U_{m} \sin \psi_{u}}{2 \omega}-\frac{D_{4} \cdot m+\omega n}{2}
\end{array}\right\} .
$$

Let's denote $m$ and $n$ in steady-state modes by $m_{0}$ and $n_{0}$. Substituting their steady-state values in (19) instead

of $m$ and $n$, and equating them $\frac{d n}{d t}=0 ; \frac{d m}{d t}=0$, we obtain a system of algebraic equations:

$$
\left\{\begin{array}{l}
\frac{-D_{1} X_{m n}(0)+D_{2} M(0)-D_{3} U_{m} \cos \psi_{u}}{2 \omega}-\frac{D_{4} n(0)-\omega m(0)}{2}=0 \\
\frac{D_{1} Y_{m n}(0)-D_{2} N(0)+D_{3} U_{m} \sin \psi_{u}}{2 \omega}-\frac{D_{4} m(0)+\omega n(0)}{2}=0
\end{array}\right\} .
$$

Solving the system (20), we can find the steady-state values of the amplitudes $m_{0}$ and $n_{0}$.

$n=n_{0}+\Delta n$. Taking into account the above, we obtain Let the perturbation result in small increments of the from $(20)$ oscillation amplitudes $\Delta m$ and $\Delta n$, i.e. $m=m_{0}+\Delta m$,

$$
\left\{\begin{array}{l}
\frac{d\left(n_{0}+\Delta n\right)}{d t}=\frac{-D_{1} \cdot X_{\left(\begin{array}{c}
n+\Delta+\Delta n \\
m 0+\Delta m
\end{array}\right)}+D_{2} \cdot M_{\left(\begin{array}{c}
n+\Delta+\Delta n \\
m 0+\Delta n
\end{array}\right)}-D_{3} U m \cos \psi_{u}}{2 \omega}-\frac{D_{4}\left(n_{0}+\Delta n\right)-\omega\left(m_{0}+\Delta m\right)}{2} \\
\frac{d\left(m_{0}+\Delta m\right)}{d t}=\frac{D_{1} \cdot Y_{\left(\begin{array}{c}
n 0+\Delta n \\
m 0+\Delta n
\end{array}\right)}-D_{2} \cdot N_{\left(\begin{array}{c}
n 0+\Delta n \\
m 0+\Delta m
\end{array}\right)}+D_{3} U m \sin \psi_{u}}{2 \omega}-\frac{D_{4} *\left(m_{0}+\Delta m\right)+\omega\left(n_{0}+\Delta n\right)}{2}
\end{array}\right\}
$$

We expand the values $\frac{d\left(n_{0}+\Delta n\right)}{d t}$ and $\frac{d\left(m_{0}+\Delta m\right)}{d t}$ from the last expression to the Taylor series in small increments of $\Delta n$ and $\Delta m$, and because of their smallness, we restrict the summands with the first powers of $\Delta n$ and $\Delta m$. For the specified decomposition, we find the partial derivatives:

$$
\begin{gathered}
\frac{d X_{m n}}{d_{m}}=4,5\left(s-A_{2 m}\right)^{8} * A_{2}+137,9\left(-c-A_{2 n}\right)^{2}\left(s-A_{2 m}\right)^{6} \cdot A_{2}+ \\
+147,5\left(-c-A_{2 n}\right)^{4}\left(s-A_{2 m}\right)^{4} \cdot A_{2}+59,1\left(-c-A_{2 n}\right)^{6}\left(s-A_{2 m}\right)^{2} \cdot A_{2}+2,5\left(s-A_{2 m}\right)\left(-c-A_{2 n}\right)^{8} \cdot A_{2} \\
\frac{d X_{m n}}{d_{n}}=-39,4\left(-c-A_{2 n}\right)\left(s-A_{2 m}\right)^{7} \cdot A_{2}-118\left(-c-A_{2 n}\right)^{3}\left(s-A_{2 m}\right)^{5}- \\
-118,2\left(-c-A_{2 n}\right)^{5}\left(s-A_{2 m}\right)^{3} \cdot A_{2}-20\left(-c-A_{2 n}\right)^{7}\left(s-A_{2 m}\right) \cdot A_{2} \\
\frac{d Y_{m n}}{d_{m}}=118,4\left(-c-A_{2 n}\right)\left(s-A_{2 m}\right)^{7} \cdot A_{2}+236,4\left(s-A_{2 m}\right)^{5}\left(-c-A_{2 n}\right)^{3} \cdot A_{2}+ \\
+118\left(s-A_{2 m}\right)^{3}\left(-c-A_{2 n}\right)^{5} \cdot A_{2}+19,6\left(s-A_{2 m}\right)\left(-c-A_{2 n}\right)^{7} \cdot A_{2} \\
\frac{d Y_{m n}}{d_{n}}=-14,8\left(s-A_{2 m}\right)^{8} A_{2}-118,2\left(-c-A_{2 n}\right)^{2}\left(s-A_{2 m}\right)^{6} \cdot A_{2}- \\
-147,5\left(-c-A_{2 n}\right)^{4}\left(s-A_{2 m}\right)^{4} \cdot A_{2}-68,6\left(-c-A_{2 n}\right)^{6}\left(s-A_{2 m}\right)^{2} \cdot A_{2}-4,5\left(-c-A_{2 n}\right)^{8} \cdot A_{2} \\
\frac{d M_{m n}}{d_{m}}=4,5 m^{8}+137,9 n^{2} m^{6}+147,5 n^{4} m^{4}+59,1 n^{6} m^{2}+2,5 n^{8} . \\
\frac{d M_{m n}}{d_{n}}=39,4 m^{7} n+118 m^{5} n^{3}+118,2 n^{3} m^{5}+20 m n^{7} . \\
\frac{d N_{m n}}{d_{m}}=118,4 m n^{7}+2326,4 n^{3} m^{5}+118 n^{5} m^{3}+19,6 n^{7} m .
\end{gathered}
$$




$$
\frac{d N_{m n}}{d_{n}}=14,8 m^{8}+118,2 m^{6} n^{2}+147,5 m^{4} n^{4}+68,6 m^{2} n^{6}+4,5 n^{8} .
$$

Insert the values $m_{0}$ and $n_{0}$ of the steady state mode

into the found expressions and enter the notation

$$
\begin{aligned}
& \frac{d X_{m n(m=m 0, n=n 0)}}{d_{m}}=X_{1} ; \frac{d X_{m n(m=m 0, n=n 0)}}{d_{n}}=X_{2} ; \frac{d Y_{m n(m=m 0, n=n 0)}}{d_{m}}=Y_{1} ; \frac{d Y_{m n(m=m 0, n=n 0)}}{d_{n}}=Y_{2} ; \\
& \frac{d M_{m n(m=m 0, n=n 0)}}{d_{m}}=M_{1} ; \frac{d M_{m n(m=m 0, n=n 0)}}{d_{n}}=M_{2} ; \frac{d N_{m n(m=m 0, n=n 0)}}{d_{m}}=N_{1} ; \frac{d N_{m n(m=m 0, n=n 0)}}{d_{n}}=N_{2}
\end{aligned}
$$

We also denote

$$
X_{m n(m=m 0, n=n 0)}=X_{0} ; Y_{m n(m=m 0, n=n 0)}=Y_{0} ; M_{m n(m=m 0, n=n 0)}=M_{0} ; N_{m n(m=m 0, n=n 0)}=N_{0}
$$

Substituting expressions (21) and (22) for derivatives after transformations, we obtain a system of equations that takes into account increments

$$
\begin{aligned}
& \frac{D_{1} \cdot X_{(m 0+\Delta m ; n 0+\Delta n)}}{2 \omega}+\frac{D_{2} \cdot M_{(m 0+\Delta m ; n 0+\Delta n)}}{2 \omega}-\frac{D_{3} \cdot U_{m} \cos \psi_{u}}{2 \omega}-\frac{D_{4(n 0+\Delta n)}}{2}-\frac{\omega_{(m 0+\Delta m)}}{2}= \\
& =\frac{D_{1} X_{0}}{2 \omega}+\frac{D_{2} M_{0}}{2 \omega}-\frac{D_{3} U_{m} \cos \psi_{u}}{2 \omega}-\frac{D_{4} n_{0}}{2}-\frac{\omega m_{0}}{2}+ \\
& +\Delta m\left(\frac{D_{1} X_{1}}{2 \omega}+\frac{D_{2} M_{1}}{2 \omega}-\frac{\omega}{2}\right)+\Delta n\left(\frac{D_{1} X_{2}}{2 \omega}+\frac{D_{2} M_{2}}{2 \omega}-\frac{D_{4}}{2}\right) \\
& \text { Given that } \frac{d_{(m 0+\Delta m)}}{d t}=\frac{d_{\Delta m}}{d t}, \quad \frac{d_{(n 0+\Delta n)}}{d t}=\frac{d_{\Delta n}}{d t} \text {, } \\
& \left\{\begin{array}{l}
\frac{d_{\Delta m}}{d t}=\left(\frac{D_{1} X_{1}+D_{2} M_{1}}{2 \omega}-\frac{\omega}{2}\right) \Delta m+\left(\frac{D_{1} X_{2}+D_{2} M_{2}}{2 \omega}-\frac{D_{4}}{2}\right) \Delta n \\
\frac{d_{\Delta n}}{d t}=\left(\frac{D_{1} Y_{1}+D_{2} N 1}{2 \omega}-\frac{\omega}{2}\right) \Delta m+\left(\frac{D_{1} X_{2}+D_{2} M_{2}}{2 \omega}-\frac{D_{4}}{2}\right) \Delta n
\end{array}\right\} \\
& \alpha=\frac{D_{1} X_{1}+D_{2} M_{1}}{2 \omega}-\frac{\omega}{2} ; \beta=\frac{D_{1} X_{2}+D_{2} M_{2}}{2 \omega}-\frac{D_{4}}{2} ; \\
& \gamma=\frac{D_{1} Y_{1}+D_{2} N_{1}}{2 \omega}+\frac{\omega}{2} ; \delta=\frac{D_{1} Y_{2}+D_{2} N_{2}}{2 \omega}-\frac{D_{4}}{2} .
\end{aligned}
$$
expression (23) is written as

Denote

\section{Realization of the concept}

From (26) we make a characteristic equation, for which we express $\Delta m$ from the upper expression (26) and substitute the lower expression (26). Reducing the resulting expression by $\Delta n$, after transformations we get $P^{2}-P(\alpha+\delta)-\alpha \cdot \delta-\gamma \cdot \beta=0$

According to the Hurwitz criterion, for the attenuation of $\Delta m$ and $\Delta n$, the conditions must be met:

$$
-(\alpha+\delta)>0 ;(\alpha \cdot \delta-\gamma \cdot \beta)>0 \text { (27) }
$$

In expression (27), we replace the inequality signs with equal signs, express the value of $\alpha$ from the first expression, after substitution in the second expression, and after conversion, we get an expression for a function whose points lie on the interface between stable and unstable modes of operation of the chain

$$
\alpha=\sqrt{-\gamma \cdot \beta}
$$

To get real values of $\alpha$, it is necessary that the root expression is equal to zero or greater than zero, therefore, combinations of $\gamma$ and $\beta$ are possible only with different signs.

In the fig. 2 shows a graph of dependencies $\alpha=\sqrt{-\gamma \cdot \beta}$, which are surfaces located above the second $(S 1)$ and fourth $(S 2)$ quadrants.

Algebraize (24) and taking into account (25), we get

$$
\begin{aligned}
& P \cdot \Delta m=\alpha \cdot \Delta m+\beta \cdot \Delta n \\
& P \cdot \Delta n=\gamma \cdot \Delta m+\delta \cdot \Delta n
\end{aligned}
$$

Under 27, function points, lying under the surfaces $S 1$ and $S 2 \quad(\alpha<\sqrt{-\gamma \cdot \beta})$ are points of unstable operation of the device, the points belonging to the surfaces $S 1$ and $S 2(\alpha<\sqrt{-\gamma \cdot \beta})$ characterize the work chain on the border of stable and unstable regions, and the points lying above the surfaces $S 1$ and $S 2$ $(\alpha<\sqrt{-\gamma \cdot \beta})-$ characterize the work of the circuit in the stable area.

There are two possible modes of operation at the stability boundary:

1)when $-\gamma=\beta$ or $-\beta=\gamma$, when one value of $\alpha$ corresponds to one combination of $\gamma$ and $\beta$ (the mode point is located on the curves $O A$ or $O A^{\prime}$ ), belonging to the surfaces $S 1, S 2$, respectively, and in this combination of $\gamma$ and $\beta$ in the chain, it is impossible to change the parameters abruptly;

2)for $\gamma>-\beta$ or $\beta>-\gamma$, when one value of $\alpha^{\prime}$ corresponds to two combinations of $\gamma$ and $\beta$, and there are two points $B$ and $B$ ' of possible unstable equilibrium, and in the chain there are possible abrupt changes in parameters. 


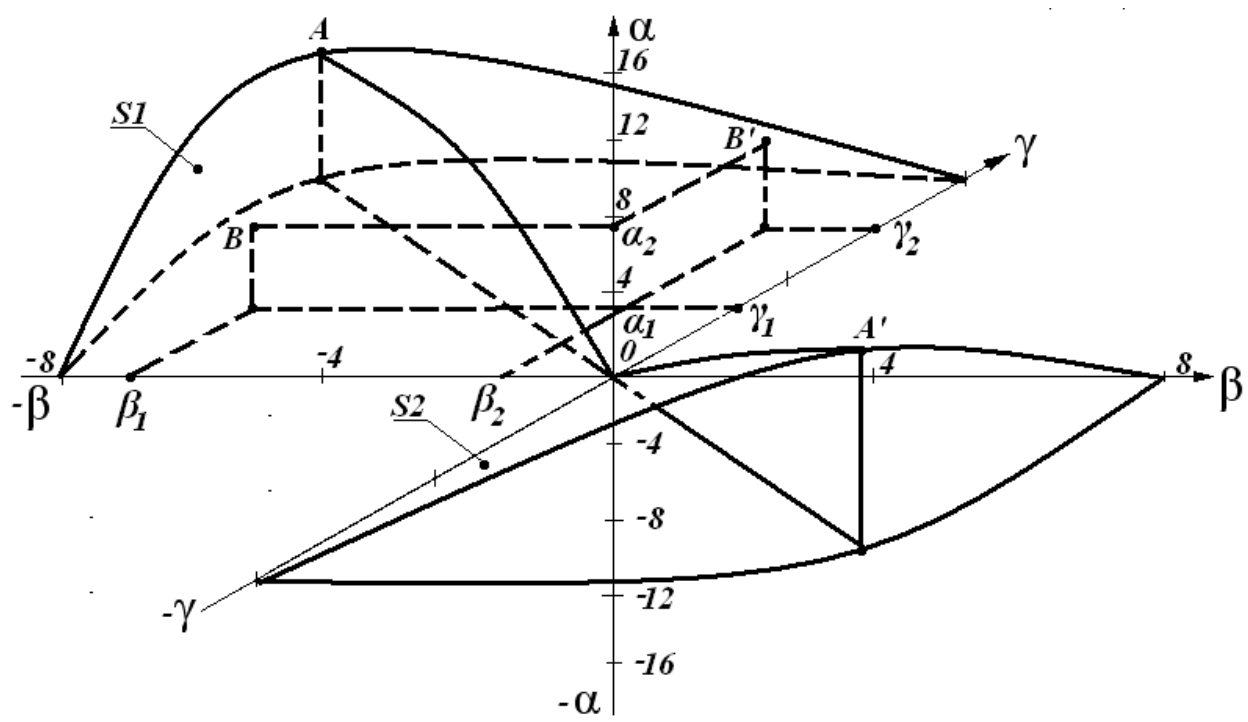

Fig.2. Graph of dependencies $\alpha=\sqrt{-\gamma \cdot \beta}$

\section{Conclusion}

1. Studies of the stability and operation of the device with the scheme in Fig.1 when the parameters $C_{1}, C_{2}=10-30 m k F, W_{1}, W_{2}=300-450 \quad$ turns, $g_{1}=0,0015 \mathrm{Om}^{-1}, g_{1}=0,0015-0,05 \mathrm{Om}^{-1}$ are varied, the results obtained on the basis of expression (28) show that the periodic process at the fundamental frequency in this circuit is stable.

2. Changing the resistances $Z_{1}, Z_{2}, Z_{3}$ of the phase number converter loads from 0 (short circuit) to $\infty$ (idle) does not affect the stability mode.

3. From the point of view of stability theory, the circuit is suitable for the development of real devices, in particular, for parametric frequency converters.

\section{References}

1. Karimov A. S., Rakhimov G. R. auto-Parametric converters of the number of phases and frequency of alternating current. Tashkent. "Fan" - 1995,- p. 275.

2. Nazarov S. L., Udintsev V. N., Bychkov S. A., Tarasov F. E., Beavogi P., Abdullaev J.O. Phase number converters in electrical engineering// Ural Federal University named after the first President of Russia B. N. Yeltsin. Yekaterinburg.- 2019.

3. Varfolomeev G. N., Mutiny S. V., Shchurov N. I., Chemodanova O. V., Chemodanova S. S., Fleisher O. N. Review of circuit solutions for phase number converters on transformers// Improvement of technical means of electric transport. Novosibirsk, 2001, pp. 78-96.

4. Evdokimov S. A. Geometric method for generating circuit solutions for phase number converters for rectifiers// Scientific Bulletin of Novosibirsk state technical University. -2008.- № 2 (31). -pp. 107-120.

5. Glushchenko V. P. Description, results of calculation and research of operating modes of some devices, electrical circuits and systems using nonlinear phenomena, reactivity, containing nonlinear components.//The author's abstract dis. ... doctor. techn. sciences/ Kharkiv Academy of Sciences. gorodskogo khoz-va. Moscow, 1997.

6. Karimov A.S., Turdiev M.T., Khudaykulov Z.R., Ibragimov N.R. Ferromagnetic frequency converter and phase number four times// author's certificate SU 1741239 A1, 15.06.1992.

7. Golubev A.N., Ignatenko S.V. Characteristics optimization for a frequency converter power filter as a function of phase number // Elektronechnic. - 1999. № 7.- c. 42-46.

8. Sidorov S.A., Roginskaya L.Z. Phase-converting device for asymmetric loads//Proceedings of electric power systems and networks. Energy saving. Ufa:.With.121-125.-2013y.

9. Bedritskiy I., Juraeva K., Bazarov L., Saidvaliev B. Using of the parametric nonlinear LC-circuitsin stabilized converters of the number of phases// Jour. of Adv Research in Dynamical \& Control Systems, Vol. 12, Issue-06, s. 98-107.-2020

10. Fayziev M.M., Beitullaev R.Kh., Norboev A.E. Questions of stability of the solution of a ferroresonance circuit with linear inductance.// Bulletin of science and education. No. 1(25), pp. 15-18.-2017

11. Danilov L.V., Matkhanov P.N., Filippov E.S. Theory of nonlinear electric circuits. L.: Energoatomizdat, 1990.-256 p. 